See discussions, stats, and author profiles for this publication at: https://www.researchgate.net/publication/269050277

\title{
Effects of long-lasting interplanetary magnetic field perturbations on the equatorial ionosphere
}

Conference Paper · August 2009

DOI: 10.1190/sbgf2009-019

CITATIONS

0

12 authors, including:

José H Sobral

National Institute for Space Research, Brazil

245 PUBLICATIONS $\mathbf{3 , 9 6 4}$ CITATIONS

SEE PROFILE

Mangalathayil Ali Abdu

National Institute for Space Research, Brazil

532 PUBLICATIONS $\mathbf{8 , 3 8 1}$ CITATIONS

SEE PROFILE
READS

26

Walter D. Gonzalez

National Institute for Space Research, Brazil

410 PUBLICATIONS $\quad \mathbf{1 0 , 3 1 0}$ CITATIONS

SEE PROFILE

Clezio M. Denardini

National Institute for Space Research, Brazil

129 PUBLICATIONS 582 CITATIONS

SEE PROFILE

Some of the authors of this publication are also working on these related projects:

Mesospheric Dynamics coupling with Ionosphere View project

Solat flare effects in the ionosphere during magnetic disturbances View project 


\section{Effects of long-lasting interplanetary magnetic field perturbations on the equatorial ionosphere}

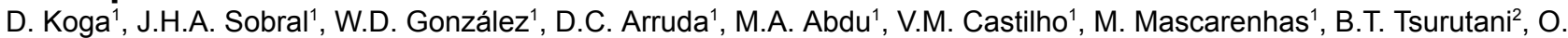
Verkhoglyadova $^{3}$, A.J. Mannucci ${ }^{2}$, C.M. Denardini ${ }^{1}$, C.J. Zamlutti ${ }^{1}$

1 National Institute for Space Research (INPE), Brazil

2 Jet Propulsion Laboratory, USA

3 IGPP, University of California, Riverside, USA

Copyright 2009, SBGf - Sociedade Brasileira de Geofísica

This paper was prepared for presentation during the $11^{\text {th }}$ International Congress of the Brazilian Geophysical Society held in Salvador, Brazil, August 24-28, 2009.

Contents of this paper were reviewed by the Technical Committee of the $11^{\text {th }}$ International Congress of the Brazilian Geophysical Society and do not necessarily represent any position of the SBGf, its officers or members. Electronic reproduction or storage of any part of this paper for commercial purposes without the written consent of the Brazilian Geophysical Society is prohibited.

\section{Abstract}

During 3 - 7 July, 2003, long-lasting and large-amplitude interplanetary magnetic field perturbation event occurred. This event is classified as high-intensity long-duration continuous AE activity (HILDCAA) which occurs outside the main phase of magnetic storms. The responses of the equatorial ionosphere to HILDCAA events have been poorly known so far. In this work, we investigate the effects of HILDCAA events on the equatorial ionosphere using ionosonde data (hmF2) observed at São Luís, Brazil and the ACE satellite data. The results indicate presence of short-time prompt penetration electric field disturbances and long-lived positive charge accumulation at the magnetic equator around the midnight sector due to the disturbance dynamo effect.

\section{Introduction}

Responses of the equatorial ionosphere to different levels of magnetic storms have been investigated for several decades (for example: Abdu, 1997; Sobral et al., 1997; Sobral et al., 2001). The equatorial ionospheric disturbances during magnetic storms are mainly generated by prompt penetration of the interplanetary electric fields (PPEF) into low-latitude from the auroral region (Kelly et al., 1979; Spiro et al., 1998; Fejer and Scherliess, 1997) and disturbance dynamo electric fields (DDEF) and neutral thermospheric winds (Blanc and Richmond, 1980; Abdu, 1997; Scherliess and Fejer, 1997; Richmond et al., 2003).

From the viewpoint of observational studies, Sobral et al. (1997) investigated an importance of the immediate previous history of the geomagnetic conditions to responses of the equatorial ionosphere. From their results we can expect the following responses of the equatorial ionosphere at sunset sector during magnetic storms (without losing generality): with quiet $\mathrm{AE}$ conditions preceding a day a large enhancement of the prereversal vertical drift velocity occurs due to a PPEF (eastward electric field) adding to the sunset F-layer dynamo (eastward electric field), while with disturbed AE conditions preceding a few to several hours an inhibition of the vertical drift enhancement is produced by the addition of a DDEF (westward electric field) to the normal evening F-layer dynamo.

Fejer and Scherliess (1997) and Scherliess and Fejer (1997) demonstrated responses of the equatorial ionosphere to moderate magnetic storms using an empirical model based on 20-year period observation of the $A E$ index and vertical plasma drift velocities from incoherent scatter radar measurement at the Jicamarca Radio Observatory. This model gives us a general view of equatorial ionospheric responses to magnetic storms. According to this model, the ionosphere can respond to PPEF input promptly ( $7.5 \mathrm{~min})$ and disturbance dynamo efficiency due to DDEF reaches to the maximum a few hours after magnetic storm onset in the post-midnight sector.

As mentioned in the above observational and theoretical investigations, we have been able to understand the behaviour of the equatorial ionosphere during magnetic storms generated by a single penetration of the interplanetary electric field, although the difficulty identifying contribution of PPEF and DDEF still remains because of modification of the thermospheric dynamics imposed by disturbance dynamo winds.

Tsurutani and Gonzalez (1987) reported high-intensity and long-lasting AE activity events a few days after the main phase of magnetic storms, that is, the recovery phase. They named this type of events as High-Intensity Long-Duration Continuous AE activity (HILDCAA) event. The main feature of the HILDCAA events is there always exist high-frequency and large IMF Bz fluctuations. The IMF Bz fluctuations can take place multiple magnetic reconnections at the dayside magnetopause, and due to the long-lasting multiple penetration of the interplanetary electric field it would significantly influence the dynamics of the entire ionosphere without any magnetic storms.

Equatorial and low-latitude ionospheric responses to HILDCAA events were reported by Sobral et al. (2006) for the first time. The influence of PPEF on the equatorial ionosphere was not clearly appeared in their cases since the HILDCAA events they selected did not show remarkable large IMF Bz fluctuations, on the other hand, they found post-sunset inhibitions of the ionospheric Flayer rise due to DDEF. Wei et al. (2008) showed the interplanetary reconnection electric field pulses can 
effectively penetrate into the equatorial ionosphere without shielding during HILDCAA period. The high value of correlation coefficient between reconnection electric field and equatorial zonal electric field was shown for a long time period ( $\sim 5$ days). The purpose of this study is to investigate minutely the effects of long-lasting IMF Bz perturbations on the equatorial ionosphere in the Brazilian sector, an extended study of Sobral et al. (2006).

\section{Experimental Data}

The ionospheric data used in this study are the F-layer electron density peak height, hmF2. They are the 15-min interval data obtained from the ionosonde station São Luís - SL (44.6W, 2.33S, dip angle 1.5S), Brazil. The definition of the HILDCAA event was empirically given by Tsurutani and Gonzalez (1987). HILDCAA consists of an auroral event such that the $A E$ index should not range below $200 \mathrm{nT}$ for more than 2 hours, should reach peaks over $1000 \mathrm{nT}$ and should occur outside the main phase of magnetic storms (the recovery phase of the magnetic storms). Furthermore, the following requisites are also the necessary in order for the auroral event (AE index variations) to be considered a HILDCAA event conditions (but not the sufficient conditions): a moderate variation of the SYM-H index, a high-speed solar wind and high frequency fluctuations IMF Bz around zero nT. In present study, the period of 21 LT 2 July - 21 LT 7 July 2003 (5 days) is selected as a HILDCAA event. In addition to this period, we analyse the previous 5 days (21 LT 27 June 21 LT 2 July) and the following 5 days (21 LT 7 July -21 LT 12 July) in order to compare to the effect of the HILDCAA event. For the sake of convenience, these data are subtracted from those of a reference day representing the average of three quiet days under criteria which the $\mathrm{Kp}$ index is less than 4- and $\Sigma \mathrm{Kp}$ is less than $24+$ during a 20-day interval centered on the HILDCAA period, i.e., $\Delta \mathrm{hmF2}=\mathrm{hmF2}$ (Disturbed day) - hmF2avg (Quiet day reference average).

We use the solar wind data observed by the ACE satellite, the solar wind velocity Vsw km/s (64-sec sampling rate) and the interplanetary magnetic field (IMF), Bx, By, Bz nT (16-sec sampling rate) in the geocentric solar magnetospheric (GSM) coordinate system. The ACE satellite is orbiting around the L1 libration point and the ACE data are distributed by the CDAWeb of the NASA (http://cdaweb.gsfc.nasa.gov/). One of the most important interplanetary parameters is the interplanetary reconnection electric field. The electric field is related to reconnection processes at the dayside magnetopause and is dawn-to-dusk (dusk-to-dawn) direction when the IMF Bz is southward (northward). Using the ACE data, the electric field is derived by assuming that the solar wind plasma motion is determined by $E \times B$ drift. Then the interplanetary reconnection electric field is $E_{R}$ $=\mathrm{Vx}\left(\mathrm{By}^{2}+\mathrm{Bz}^{2}\right)^{1 / 2} \sin (\theta / 2)$ where $\theta$ is the IMF clock angle in the $Y-Z$ plane in the GSM coordinate system, with 0 deg and 180 deg indicating northward and southward IMF, respectively. This electric field consists of two components, i.e., parallel and transverse components to the Earth magnetic field. The former contributes to the acceleration of charged particles along the magnetic field lines at the magnetopause while the later becomes the origin of the polar cap potential drops or auroral electrojet. The later is thus an important parameter for the study of the electrodynamics of the ionosphere. The transverse component of the reconnection electric field is derived from $E_{\text {perp }}=E_{R} \sin (\theta / 2)$ measured in $\mathrm{mV} / \mathrm{m}$ (Gonzalez and Mozer, 1974; Kan and Lee, 1979). Hereafter $E_{\text {perp }}$ is described as $E_{R}$ for the sake of simplicity. The time shift of the ACE data is determined as follows. We assume the distance between the ACE and the mangetopause, $L$ $1.4 \times 10^{5} \mathrm{~km}$, and using the average solar wind velocity $<$ Vsw $>$ during the period of interest the propagation delay is calculated as $T \sim L /<V$ sw $>$. In order to check the magnitude of geomagnetic activity the AE, SYM-H and Kp indices (1-min sampling rate) are used, which are obtained from the World Data Center for Geomagnetism, Kyoto (http://swdcwww.kugi.kyoto-u.ac.jp/index.html). For the purpose of comparing the ACE data with the ionospheric data, the ACE data are resampled to 1-sec value using a linear interpolation, then the $V s w, B z, E_{R}$, $A E$, and SYM-H data are averaged from $t-15 \mathrm{~min}$ to $t$, i.e., $15-\mathrm{min}$ averaged data. Furthermore, in order to compare theoretical predictions with observations, it is used an empirical model describing the average characteristics of the equatorial vertical plasma drifts developed by Fejer and Scherliess (1997) for PPEF effects and Scherliess and Fejer (1997) (hereafter FS97) for DDEF effects.

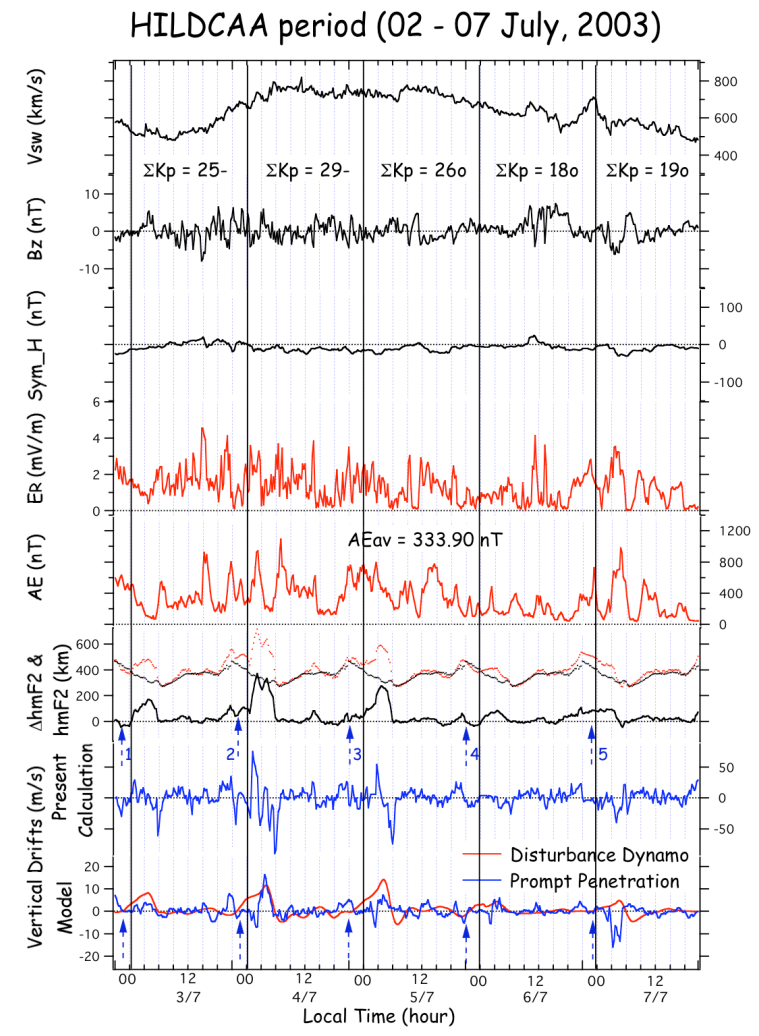

Figure 1: Interplanetary (Vsw, Bz, $\left.E_{R}\right)$, geomagnetic (AE, SYM-H) and ionospheric F-layer (hmF2, $\Delta \mathrm{hmF2}$ ) parameters. The bottom two panels show plasma vertical drifts calculated from present observation and FS97 empirical model. 


\section{Results and Discussion}

In this section, we show case studies of the equatorial ionospheric responses at São Luís for three periods: before ( 27 June -2 July), during ( 2 July -7 July) and after ( $7-12$ July) the HILDCAA event in 2003. We should note that before the HILDCAA period the variation of the ACE parameters and geomagnetic indices are similar to the definition of the HILDCAA, on the other hand, a strong magnetic storm occurred during the period after the HILDCAA event (Dst < -100 nT). In this report we focus on the results during the HILDCAA period.

HILDCAA event: 2 - 7 July 2003

Solar wind parameters such as solar wind velocity Vsw, $\mathrm{Bz}, \mathrm{E}_{\mathrm{R}}$, and the SYM-H index are presented from the first to fourth panel, respectively in Fig. 1. During these 5 days the solar wind velocity reaches the maximum value ( $800 \mathrm{~km} / \mathrm{s}$ ) on 4 July and declines gently to the minimum value $(\sim 500 \mathrm{~km} / \mathrm{s})$ on 7 July. The SYM-H index remains a steady state and does not decrease less than $\sim-50 \mathrm{nT}$. This indicates there is no significant magnetic storm (the recovery phase of a magnetic storm) in the magnetosphere. The IMF Bz fluctuates remarkably while the solar wind velocity is high. Accompanied by this large and long-lasting fluctuation of the IMF Bz, $E_{R}$ demonstrates multiple reconnection processes at the dayside magnetopause. In fifth panel of Fig. 1, the $A E$ index reaches to approximately $1200 \mathrm{nT}$ where the average value of the AE index is $333.90 \mathrm{nT}$, and satisfies the condition of HILDCAA event. The correlation between the $A E$ index and $E_{R}$ is approximately 0.64 (see Fig. 3). It indicates that the penetration efficiency of the interplanetary reconnection electric field into the auroral region is high. The sixth panel of Fig. 1 represents hmF2 (red dot), hmF2avg (black dot), and $\Delta \mathrm{hmF2}$ (black solid). The quiet day value hmF2avg was estimated using hmF2 data of 3 quiet days: 21LT 19 June $-21 \mathrm{LT} 20$ June $(\Sigma \mathrm{Kp}$ = 20o), 21LT 30 June -21 LT 1 July $(\Sigma K p=16+), 21$ LT 7 July -21 LT 8 July $(\Sigma K p=5+)$ in 2003 . During the daytime the variation of hmF2 does not differ from the quiet day level. On the nighttime, from 00 LT to 06 LT a rise of the F-region peak height is shown all the days. As expected from the results of Sobral et al. (1997), when AE conditions preceding hours are disturbed (relatively quiet), an inhibition (enhancement) of prereversal enhancement is seen around $18 \mathrm{LT}$.

In seventh and eighth panel of Fig. 1, calculated and modelled (FS97) vertical plasma drifts due to PPEF (blue line) and DDEF (red line) are shown. The calculated vertical drifts are derived as the average of two velocities: $\mathrm{v} 1=(\mathrm{hmF} 2(\mathrm{t})-\mathrm{hmF} 2(\mathrm{t}-15 \mathrm{~min})) / \mathrm{dt}, \mathrm{v} 1=(\mathrm{hmF} 2(\mathrm{t})-\mathrm{hmF} 2(\mathrm{t}-$ $30 \mathrm{~min})) / \mathrm{dt}, \mathrm{v}=(\mathrm{v} 1+\mathrm{v} 2) / 2$. We consider this velocity due to PPEF because of the short-time oscillation. The calculated and modelled vertical velocities are not as good as we expected. However, we can partially see agreement between them, e.g., 15 LT on July 3 and $~ 03$ LT on July 7 . The calculated velocity would be modified due to other factors such as coupling effects between PPEF and DDEF (Maruyama et al., 2005), occurrence of substorm-like disturbance. Indeed, there exist injection events in the magnetotail during the HILDCAA period (not shown here).
Next, comparing $\triangle \mathrm{hmF} 2$ with DDEF variation, one can see a good agreement of eastward turning of the zonal electric fields (increase of $\Delta \mathrm{hmF} 2$ ) around $2230 \mathrm{LT}$ shown by the arrows. The rise of peak height is mainly related to the reversal of the equatorial zonal electric field at the night sector. Huang et al. (2005) demonstrated local time dependence of reversal of the disturbance dynamo electric field using the NCAR TIEGCM numerical model. The simulation implies the reversal of DDEF occurs approximately 2230 LT at São Luís. Thus, our result is consistent with the simulation results of Huang et al. (2005) and indicates that positive charge accumulation at the magnetic equator around midnight sector continue to exist during the HILDCAA period.
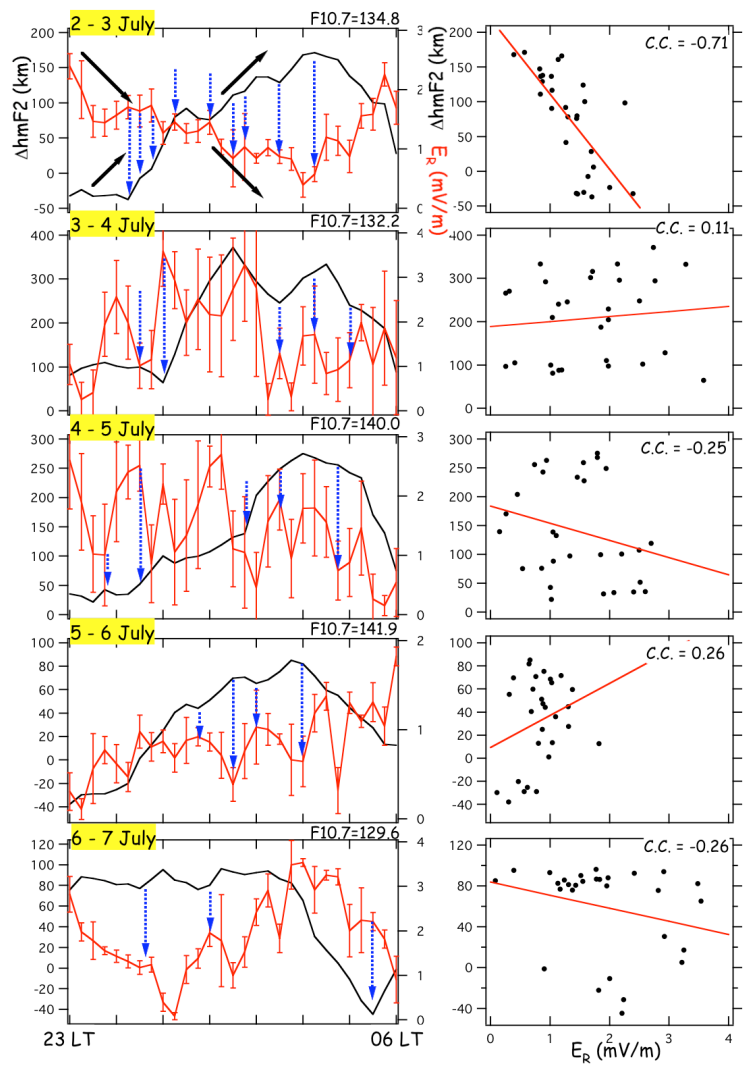

Figure 2: Correlation between $\Delta \mathrm{hmF} 2$ and $\mathrm{E}_{\mathrm{R}}$ for the nighttime (23-06 LT) during the HILDCAA period. The blue arrows in the left panels indicate negative correlation between two parameters. The abbreviation "C.C." in the right panels indicates correlation coefficients between $\Delta \mathrm{hmF} 2$ and $\mathrm{E}_{\mathrm{R}}$.

In Fig. 2, it is shown the effects of prompt penetration electric fields on the night side equatorial ionosphere between $E_{R}$ and $\Delta h m F 2$. The arrows and vertical lines in left panels indicate negative correlation between $\mathrm{E}_{\mathrm{R}}$ and $\Delta$ $h m F 2$ and the standard deviation of $E_{R}$, respectively. During the nighttime it is expected that the ionospheric altitudes respond to the interplanetary electric fields negatively, that is, $\Delta \mathrm{hmF} 2$ decreases due to the entrance of dawn-to-dusk (westward) electric fields. Indeed, there are many negative correlations between $E_{R}$ and $\Delta h m F 2$ although they are not perfect negative correlations due to the effect of DDEF. Such a short-time ( $<\sim 30$ minutes) 
prompt penetration of electric fields has never been reported so far. Thus this result will be an important progress for understanding of the equatorial ionospheric responses.

Finally, correlation coefficients between the AE index and $E_{R}$ before, during and after the HILDCAA event are shown in Fig. 3. For the upper panels the coefficients are calculated for all the 5-day period, on the other hand, the coefficients in the bottom panels are for the nighttime (23LT - 06 LT). The results indicate that the coupling between the interplanetary space and magnetosphereionosphere increases during the HILDCAA period. Since HILDCAA events maintain continuous energy input from the interplanetary space into the magnetosphere, it would be natural that the coupling force becomes stronger than other periods. Indeed, the rises of peak height before and after the HILDCAA event are less than that of the HILDCAA event (not shown here).

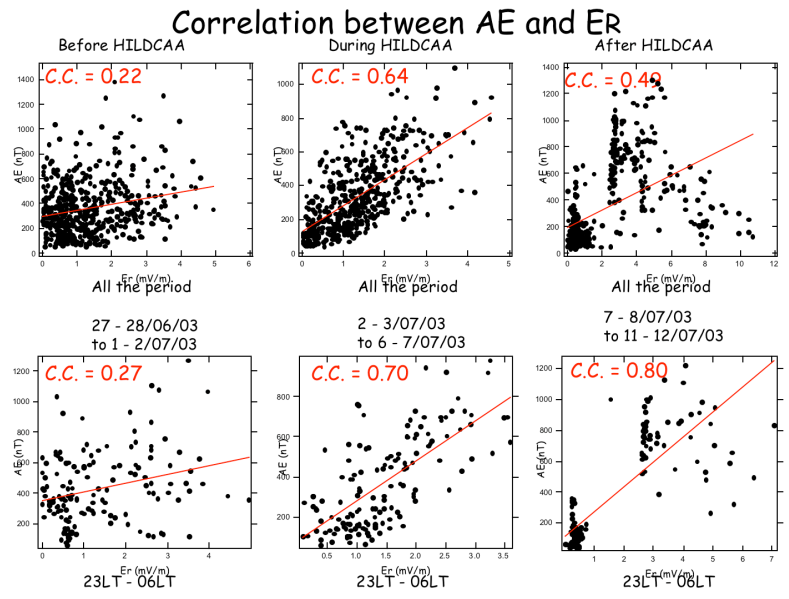

Figure 3: Correlation between the $A E$ index and $E_{R}$ for all the period (top panels) and during the night time (23-06 LT; bottom panels ), before, during and after the HILDCAA event (right to left).

\section{Conclusions}

The main conclusions of this work are the following:

Evidences of the prompt penetration electric fields during several nights of a HILDCAA event at São Luís were detected by several remarkable sequences of a negatively correlated variations of $\Delta \mathrm{hmF} 2$ both with the $A E$ index (not shown) and the interplanetary reconnection electric field $E_{R}$ and a positively correlated variation with $\mathrm{Bz}$ ( $\mathrm{Bz}$ being positive to north, not shown). Furthermore, the entrance of the interplanetary reconnection electric field into the equatorial ionosphere occurred rapidly ( 30 $\min )$.

However an overall negative correlation between $\Delta \mathrm{hmF} 2$ and $E_{R}$ for the whole night period (23LT to $06 \mathrm{LT}$ ) has not been observed on all 5 nights. Such day-to-day variation of the overall correlation may be attributed to day-to-day variations of local time thermal wind intensities and/or disturbance dynamo electric fields.
The overall correlation coefficients for the periods before, during and after the HILDCAA periods are found to be $0.22,0.64$ and 0.49 , respectively. The higher value indicates a more efficient coupling between the interplanetary medium and the earth's magnetosphere.

The over all coefficients between $\Delta \mathrm{hmF} 2$ and the parameters $A E$ index, $E_{R}$ and $B z$ each of the 5 nights during the HILDCAA period are consistent with each other.

The F-region peak height enhancement during the nighttime, approximately after $2230 \mathrm{LT}$, indicates that positive charge accumulation at the magnetic equator around midnight sector continue to exist during HILDCAA period, as demonstrated by Huang et al. (2005).

\section{Acknowledgments}

This work was supported by the Brazilian National Council for Scientific and Technological Development (CNPq) grants 152154/2007-8. We acknowledge the CDAWeb (NASA) for access to the ACE data. The SYM$\mathrm{H}, \mathrm{AE}, \mathrm{Kp}$ indices were provided by the World Data Center for Geomagnetism (Kyoto University). The F10.7 solar flux data were obtained from the NGDC (NOAA). The authors thank Maria Goreti de Santos Aquino for assistance in processing the ionosode data.

\section{References}

Abdu, M.A., 1997, Major phenomena of the equatorial ionosphere-thermosphere system under disturbed condition, Journal of Atmospheric and Solar-Terrestrial Physics, 59, 1505-1519.

Blanc, M., Richmond, A.D., 1980, The ionospheric disturbance dynamo. Journal of Geophysical Research 85, 1669.

Fejer, B.G., Scherliess, L., 1997, Storm time dependence of equatorial disturbance dynamo zonal electric field. Journal of Geophysical Research 102, 24037.

Gonzalez, W., Mozer, F., 1974, A Quantitative Model for the Potential Resulting from Reconnection with an Arbitrary Interplanetary Magnetic Field. Journal of Geophysical Research, 79(28), 4186-4194.

Huang, C.M., Richmond, A.D., Chen, M.-Q., 2005, Theoretical effects of geomagnetic activity on low-latitude ionospheric electric fields. Journal of Geophysical Research 110, A05312, doi:10.1029/2004JA010994

Kan, J. R., Lee, L. C., 1979, Energy Coupling Function and Solar Wind-Magnetosphere Dynamo. Geophysical Research Letters, 6(7), 577-580.

Maruyama, N., Richmond, A.D., Fuller-Rowell, T.J., Codrescu, M.V., Sazykin, S., Toffoletto, F.R., Spiro, R.W., Millward, G.H., 2005, Interaction between direct penetration and disturbance dynamo electric fields in the 
storm-time equatorial ionosphere. Geophysical Research Letters 32, L17105,

doi:10.1029/2005GL023763.

Richmond, A.D., Peymirat, C., Roble, R.G., 2003, Longlasting disturbances in the equatorial ionospheric electric field simulated with a coupled magnetosphere-

ionosphere-thermosphere model. Journal of Geophysical Research 108, 1118, doi:10.1029/2002JA009758.

Scherliess, L., Fejer, B.G., 1997, Empirical models of storm time equatorial zonal electric fields. Journal of Geophysical Research 101, 24047.

Sobral, J.H.A., Abdu, M.A., Gonzalez, W.D., Tsurutani, B.T., Batista, I.S., Gonzalez, A.L.C., 1997, Effects of intense storms and substorms on the equatorial ionosphere/thermosphere system in the American sector from ground-based and satellite data. Journal of Geophysical Research 102, 14305.

Sobral, J.H.A., Abdu, M.A., Yamashita, C.S., Gonzalez, W.D., Gonzalez, A.C., Gonzalez, A.C., Batista, I.S.,

Zamulutti, C.J., Tsurutani, B.T., 2001, Responses of the low-latitude ionosphere to very intense geomagnetic storms. Journal of Atmospheric and Solar-Terrestrial Physics, 63, 965-974.

Sobral, J.H.A., Abdu, M.A., Gonzalez, W.D., Gonzalez, A.C., Tsurutani, B.T., da Silva, R.R.L., Barbosa, I.G., Arruda, D.C.S., Denardini, C.M., Zamlutti, C.J., Guarnieri F., 2006, Equatorial ionospheric responses to high-intensity long-duration auroral electrojet activity (HILDCAA). Journal of Geophysical Research 111, A07S02, doi:10.1029/2005JA011393.

Spiro, R.W., Wolf, R.A., Fejer, B.G., 1988, Penetration of high latitude electric field effects to low latitudes during SUNDIAL 1984, Annales Geophysicae, 6, 39 - 50.

Tsurutani, B.T., Gonzalez, W.D., 1987, The cause of high-intensity long-duration continuous AE activity (HILDCAAS): interplanetary Alfven wave trains. Planetary and Space Science 35, 405.

Wei, Y., Hong, M., Wan, W., Du, A., Lei, J., Zhao, B., Wang, W., Ren, Z., Yue, X., 2008, Unusually long lasting multiple penetration of interplanetary electric field to equatorial ionosphere under oscillating IMF Bz. Geophysical Research Letters 35, L02102, doi:10.1029/2007GL032305. 


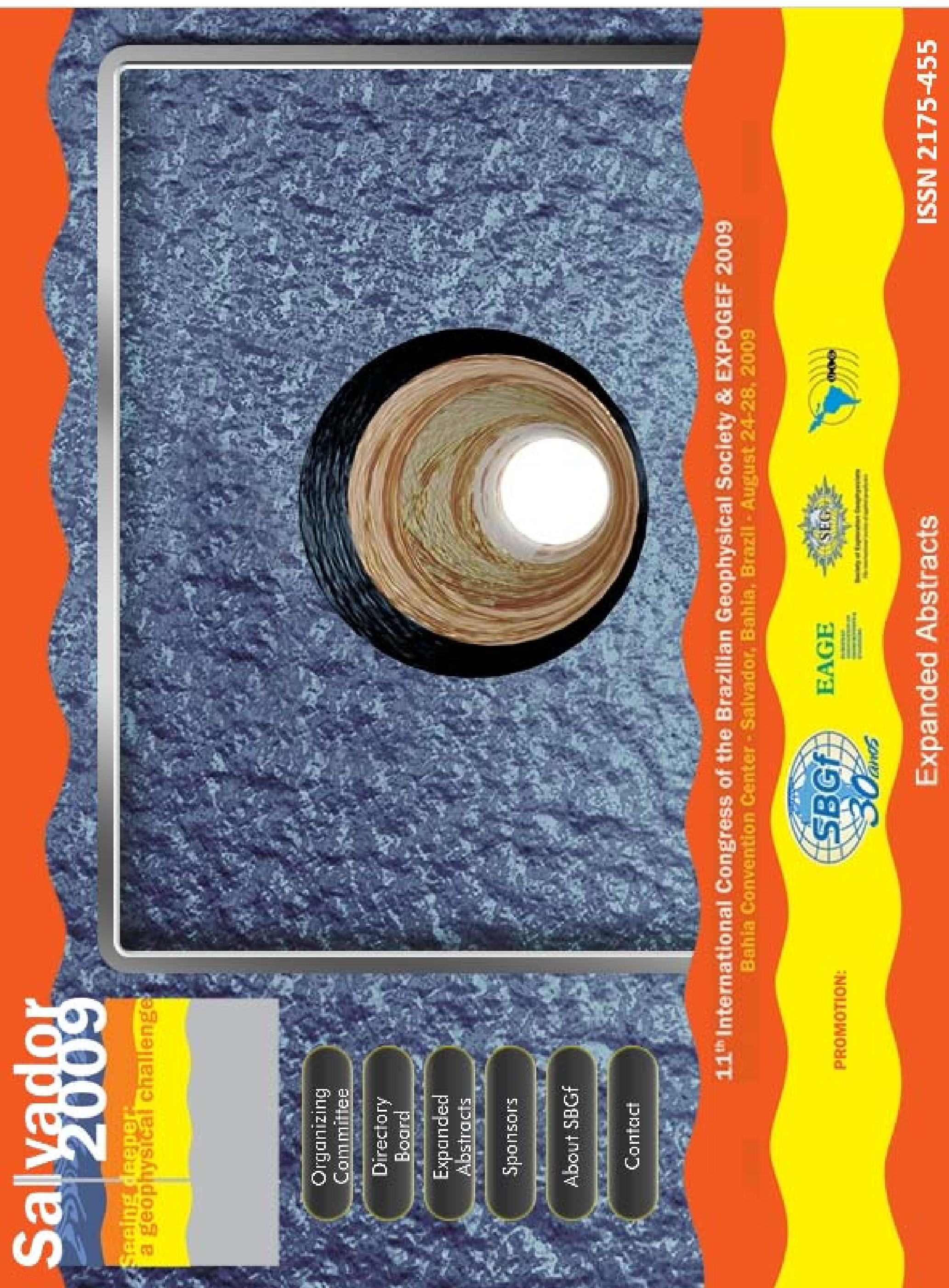

